\title{
Establishing an adequate dose of acupuncture is essential for clinical trial studies
}

\author{
Sheng-Xing Ma* \\ Lundquist Institute for Biomedical Innovation at Harbor-UCLA Medical Center and Department of Obstetrics and Gynecology, David Geffen School of Medicine \\ at University of California at Los Angeles and Harbor-UCLA Medical Center, Torrance, CA 90502, USA
}

\begin{abstract}
Importance: A number of recent clinical trials have demonstrated that acupuncture is more effective for treating chronic pain conditions compared to sham and no acupuncture, but some research questions have remained unaddressed and are standing in the way of further progress.

Observations and Advances: The effectiveness of acupuncture for pain conditions compared to usual care have been demonstrated, which has significantly enhanced the position of acupuncture in multiple pain guidelines following these studies. However, the studies also generated some conflicting results with difficulty in comparing each other. Research examining an adequate dose of acupuncture therapy with optimal intervention parameters and time table has also long been neglected and is now urgent. The dose of acupuncture depends on stimulation parameters: force/intensity and speed/frequency of manual acupuncture (MA) or electroacupuncture (EA) and time table (number of treatment sessions and duration). Various frequencies and intensities of MA and EA stimulation have been utilized in individual research. Different acupuncture treatment sessions (once, twice, three to five times per week) and periods (4, 6, 8 , and 12 weeks) have been used in these clinical trials. One clinical trial using one session of needle acupuncture and laser at 12 weeks (8 to 12 treated sessions) did not improve pain in patients with chronic knee pain but similar trials of osteoarthritis knee have significant effects of pain improvement after biweekly sessions of needle acupuncture for 8 weeks of treatment.
\end{abstract}

Conclusions and Relevance: Determining a right treatment regimen on correct acupuncture point(s) for acupuncture is a critical first step for acupuncture clinical trials. Appropriate acupuncture parameters such as acupuncture stimulation technique, treatment sessions, and treatment duration must be considered in acupuncture clinical trials. An adequate dose of acupuncture for clinical trials should be established following dose finding workshops for acupuncture before the studies, which not only improve the therapeutic effects of the therapies but also allow the comparisons between trials and between the acupuncture community/practice and trial studies.

\section{Introduction}

Over the past two decades, a number of clinical trials [1-5] and patient-level meta-analyses [6-9] on the effectiveness of acupuncture for pain conditions compared to usual care have been published in high quality journals. It is believed that the research has significantly enhanced the position of acupuncture in multiple pain guidelines and subsequent policy decisions, which can portray acupuncture for pain control at a new position of strength [10]. In contrast to these positive results, one clinical trial generated negative results and identified no such consistent effects on chronic pain [11]. A large meta-analysis of randomized clinical trials with data from 17,922 patients with chronic pain have also demonstrated that the effects of acupuncture on pain improvement have significant, but small, differences compared to sham acupuncture procedures and no difference between acupuncture points and non-points [9]. These conclusions puzzled the acupuncture community and made confusion between acupuncture practices and trial studies. Published papers questioned whether the correct acupuncture methodology was used in the trials [12]. Langevin and Wayne (2018) in a review paper pointed out that "This is a perfect time, then, to examine some research questions that are central to the field of acupuncture, but have remained mostly unaddressed and are standing in the way of further progress [10]."

The term acupuncture describes a family of procedures involving the stimulation of points on the body using a variety of techniques. Langevin and Wayne (2018) indicated that the subject of whether- or-not acupuncture points "exist" has been given too little attention within the acupuncture research community, and failure to rigorously investigate the subject of acupuncture points has hindered the growing legitimacy of acupuncture as an evidence-based therapy [10]. In addition to research on acupuncture point specificity and correct selection of the point(s), it is believed that establishing appropriate acupuncture dose and the guideline for consultation with evidence-based information to support determining an adequate dose and acupuncture points are critical important for acupuncture clinical trials [13].

Clinical trial was initiated to determine whether new drugs or treatments are both safe and effective. Knowledge of the relationships among dose, drug concentration in blood, and clinical response (effectiveness and undesirable effects) is critical important for determining the safe and effective use of drugs in individual patients [14]. Therefore, determination of a dosage is one of the first steps in drug clinical trials. In fact, dose finding workshops for new drug studies are frequently held internationally. The Guideline for Industry Dose-

${ }^{*}$ Correspondence to: Sheng-Xing $\mathrm{Ma}$, Professor, Lundquist Institute for Biomedical Innovation at Harbor-UCLA Medical Center and Department of Obstetrics and Gynecology, David Geffen School of Medicine at UCLA and Harbor-UCLA Medical Center 1124 W. Carson Street, E6-room 129, Torrance, CA 90502, USA, Tel: (310) 974-9573; E-mail: sxma@lundquist.org

Received: March 03, 2020; Accepted: March 13, 2020; Published: March 16 2020 
Response Information to Support Drug Registration was developed within the Expert Working Group (Efficacy) of the International Conference and has been supported for consultation to determine a right dose, in accordance with the Harmonization of Technical Requirements for the Registration of Pharmaceuticals for Human Use process [15]. However, if acupuncture treatment is considered as a drug in clinical trial studies, what is the right regimen of acupuncture? Is there a guideline that can be used for consultation to determine acupuncture dose for clinical trial studies?

The clinical response to acupuncture depends on stimulation parameters and time table (treatment sessions and periods), which together serve as the "dose" of acupuncture. The clinical response of electroacupuncture (EA) and manual acupuncture (MA) depends on stimulation parameters, which include force and speed for MA and frequency and intensity for EA [14,16-18]. Currently, various force/intensity and speed/frequency of MA/EA have been utilized in acupuncture clinical trials [1-5]. White, et al. have indicated that many different styles of acupuncture practices exist, and lack of agreement on the optimal acupuncture treatment for any particular condition makes the negative results in some subjects and sham controlled trials difficult to interpret [19]. They emphasized that there is an urgent need for studies to guide decisions about what is an 'adequate' dose of acupuncture and to explore whether there is evidence of a doseresponse relationship. However, there is no systematic study in humans to determine an 'adequate (optimal)' dose of acupuncture by testing various stimulating frequencies, intensities, and treatment periods for different clinical conditions. It is recommending the concept of 'dose' of acupuncture needling should include both the stimulus given to the patient (the physical procedures applied in each session), and certain aspects of the patient's perceptions (sensory, affective and cognitive), for example, the "de qi" sensation, and response that are known to be linked to the subsequent therapeutic response [20,21]. The "de qi" sensations in subjects have been evaluated by using a questionnaire containing five individual visual analog scales [21-23]. The concept of acupuncture 'dose' administered to the patient (the stimulation parameters, treatment sessions, and periods of acupuncture) is analyzed and discussed as follows.

\section{The relationships between clinical response and stimulation parameters of acupuncture}

Acupuncture techniques, as described in traditional Chinese medicine, involve a family of procedures with various methods such as lifting and thrusting the needle as well as twisting and rotating the needle by different force, speed and amplitude. The methods are typically divided into a reinforcement method attained by slowly twisting/rotating the needle with gentle force, a reduction method, attained by rapidly twisting/rotating the needle with great force, and normal reinforcement and normal reduction, which lift and thrust or twist and twirl the needle evenly causing mild sensations and withdraw the needle with moderate speed [20,24]. Reinforcement results in local feeling of warmness (vasodilation), but reduction causes local feeling of coldness. Although acupuncture carries a long history, the biological effects behind reinforcement/reduction methods are unclear.

Recently, the effects of reinforcing method using MA vs. reductive EA on local nitric oxide (NO) release have been examined using a novel biocapture device over skin regions in humans [25]. Results show that NO levels biocaptured over the skin regions are increased following MA by twisting/rotating the needle with gentle amplitude and moderate speed. In contrast, NO levels over the areas of the skin regions are moderately reduced by high-frequency EA $(30 \mathrm{~Hz})$, a reduction method. Consistently, NO levels biocaptured over the skin regions are elevated by either electrical heat and transcutaneous electrical nerve stimulation (TENS) with low-frequency $(6 \mathrm{~Hz})[25,26]$. The results suggest that heating and MA/TENS with low stimulating force and rate, which are reinforcement methods, produce an elevation of NO release predominantly over acupoints, but EA with a higher stimulating force and rate, which are reduction methods, cause an inhibition of NO generation. It is well-documented that NO improves local circulation and allows for a flush of analgesic or sensitizing substances for pain relief [27-29]. Acupuncture induces an elevation of vasodilator (NO) release over skin regions, and elevated NO improves local circulation, which contribute to local warmness and the beneficial effects of acupuncture such as pain relief, improvements of sweating, and inflammation. These results suggest that local NO release is critically dependent on the stimulating force/intensity and speed/ frequency of MA/EA, and utilizing the appropriate parameters for an investigated technique carries significant clinical relevance to conduct meaningful acupuncture research $[25,26]$.

\section{The relationships among clinical response and treatment sessions, and periods}

Various acupuncture treatment sessions (once, twice, three times, and five times per week) have been used in acupuncture clinical trials for a total length (periods) of 4, 6, 8, and 12 weeks [1-5]. A recent clinical trial has reported that needle acupuncture and laser acupuncture with 8 to 12 treated sessions during period of 12 weeks (less than once per week) do not improve pain in patients with chronic knee pain [11]. Similar acupuncture clinical trials of osteoarthritis knee have significant effects in pain after biweekly sessions of needle acupuncture for 8 weeks of treatment $[1,2,4]$. Acupuncture trials with data from 1162 patients with chronic low back pain indicated that ten 30-minute sessions of acupuncture, administered biweekly, resulted in significant improvements on pain relief but the significance was small when compared to sham acupuncture [5]. These previous studies have suggested that the effect of acupuncture on pain relief is largely dependent on acupuncture time table (treatment sessions per week and total treatment periods) in addition to stimulating frequency and intensity $[1-5,11]$. In view of the conflicting findings and small or negative results from acupuncture clinical trials, defining scientific based acupuncture time table (sessions and total length) is critically important when conducting clinical trials.

\section{Conclusion}

The dose of acupuncture stimulus given to the patient differs from a dose of drug and depends on correct acupuncture points, stimulation parameters (force/intensity and speed/frequency) and time table (treatment sessions and total length of treatment). Although many acupuncture clinical trials have been conducted and published, there is no systematic study in humans to identify the optimal parameters of MA/EA and time tables. These factors influence clinical response by patients and possibly explain the differences experienced by practicing acupuncturists in the community and the results published in acupuncture clinical trials. Determination of a clinically effective, therapeutic dose is not only an essential criterion in any drug clinical trials, but it is also very important for acupuncture clinical trials. Establishing gold-standard MA and EA therapies with correct acupuncture points, optimal intervention parameters and time table will significantly improve the therapeutic effects of the therapies and allow the comparisons between trials and between the acupuncture 
community/practice and trial studies. The guideline for consultation by acupuncture dose information and regulatory parties to support determining an adequate dose of acupuncture for clinical trials should been established following dose finding workshops for MA and EA.

\section{Funding}

This project was made possible by NIH Grant (AT002478, AT004620, and AT004504) from the National Center for Complementary and Integrative Health (to SXM).

\section{Conflicts of interest}

The author has stated explicitly that there are no conflicts of interest in connection with this article.

\section{References}

1. Berman BM, Lao L, Langenberg P, Lee WL, Gilpin AM, et al. (2004) Effectiveness of acupuncture as adjunctive therapy in osteoarthritis of the knee: a randomized controlled trial. Ann Intern Med 141: 901-910. [Crossref]

2. Witt C, Brinkhaus B, Jena S (2005) Acupuncture in patients with osteoarthritis of the knee. A randomised trial. Lancet 366: 136-143.

3. Brinkhaus B, Witt CM, Jena S (2006) Acupuncture in patients with chronic low back pain: A randomized controlled trial. Arch Intern Med 166: 450-457.

4. Berman BM, Singh BB, Lao L, Langenberg P, Li H, et al. (1999) A randomized trial of acupuncture as an adjunctive therapy in osteoarthritis of the knee. Rheumatology 38: 346-354.

5. Haake M, Müller HH, Schade-Brittinger C (2007) German acupuncture trials (GERAC) for chronic low back pain: randomized, multicenter, blinded, parallel-group trial with 3 groups. Arch Intern Med 167: 1892-1898.

6. Vickers AJ, Cronin AM, Maschino AC, Lewith G, MacPherson H, et al. (2012) Acupuncture for chronic pain: individual patient data meta-analysis. Arch Intern Med 172: 1444-1453. [Crossref]

7. MacPherson H, Vertosick E, Lewith G (2014) Influence of control group on effect size in trials of acupuncture for chronic pain: A secondary analysis of an individual patient data meta-analysis. PLoS One 9: e93739.

8. Manheimer E1, White A, Berman B, Forys K, Ernst E (2005) Meta-analysis: acupuncture for low back pain. Ann Intern Med 142: 651-663. [Crossref]

9. MacPherson H, Maschino AC, Lewith G (2013) Characteristics of acupuncture treatment associated with outcome: an individual patient meta-analysis of 17,922 patients with chronic pain in randomised controlled trials. PLoS One 8: e77438.

10. Langevin HM, Wayne PM (2018) What is the point? The problem with acupuncture research that no one wants to talk about. J Altern Complement Med 24: 200-207. [Crossref]

11. Hinman RS, McCrory P, Pirotta M, Relf I, Forbes A, et al. (2014) Acupuncture for chronic knee pain: a randomized clinical trial. JAMA 312: 1313-1322. [Crossref]

12. Li YM (2013) Puzzles and hypotheses of acupuncture. Chin J Integr Tradit West Med 33: $1445-1448$.
13. Huo RL, Ma SX (2016) Analysis of the puzzle between acupuncture community and acupuncture clinical trials. Chin J Integr Tradit West Med 36: 261-265.

14. Thall PF, Russell KE (1998) A strategy for dose-finding and safety monitoring based on efficacy and adverse outcomes in phase I/II clinical trials. Biometrics 54: 251-264.

15. Federal Register (1994) Guideline for Industry Dose-Response Information to Support Drug Registration 59: 55972-55976.

16. Foster JM, Sweeney BP (1987) The mechanisms of acupuncture analgesia. British $J$ Hospital Med 38: 308-312.

17. Han JS (2003) Acupuncture: neuropeptide release produced by electrical stimulation of different frequencies. Trends Neurosci 26: 17-22.

18. Ma SX (2004) Neurobiology of acupuncture: Toward CAM. Evid Based Complement Alternat Med 1: 41-47.

19. White A, Cummings M, Barlas P, Cardini F, Filshie J, et al. (2008) Defining an adequate dose of acupuncture using a neurophysiological approach--a narrative review of the literature. Acupunct Med 26:111-120.

20. Beijing College of Traditional Chinese Medicine (1980) Shanghai College of Traditional Chinese Medicine, Nanjing College of Traditional Chinese Medicine, The Acupuncture Institute of the Academy of Traditional Chinese Medicine. Essentials of Chinese Acupuncture. Foreign Languages Press, Beijing, pp: 301-310.

21. Kong J, Gollub R, Huang T (2007) Acupuncture De Qi, from qualitative history to quantitative measurement. J Altern Complement Med 13: 1059-1070.

22. Kou W, Gareus I, Bell JD (2007) Quantification of DeQi sensation by visual analog scales in healthy humans after immunostimulating acupuncture treatment. Am J Chin Med 35: 753-765.

23. Zhou K, Fang J, Wang X, Wang Y, Hong Y, et al. (2011) Characterization of de qi with electroacupuncture at acupoints with different properties. $J$ Altern Complement Med 17: 1007-1013. [Crossref]

24. Novey DW (2000) Clinician's Complete Reference to Complementary \& Alternative Medicine. St. Louis, MO: Mosby Inc.

25. Ma SX, Lee P, Anderson TL, Li XY, Jiang I (2017) Response of local nitric oxide release to manual acupuncture and electrical heat in humans: Effects of reinforcement methods. Evid Based Complement Alternat Med.

26. Ma SX, Mayer E, Lee P, Li XY, Gao EZ (2015) Transcutaneous electrical nerve stimulation increased nitric oxide-cyclic cGMP release biocaptured over skin surface of the pericardium meridian and acupuncture points in humans. Acup \& ElectroTherapeutics Res INT J 40: 73-86.

27. Sandberg M, Lundeberg T, Lindberg LG, Gerdle B (2003) Effects of acupuncture on skin and muscle blood flow in healthy subjects. Eur J Appl Physiol 90: 114-119. [Crossref]

28. Sandberg M, Lindberg LG, Gerdle B (2004) Peripheral effects of needle stimulation (acupuncture) on skin and muscle blood flow in fibromyalgia. Eur J Pain 8: 163 171.

29. Sandberg M, Larsson B, Lindberg LG, Gerdle (2005) Different patterns of blood flow response in the trapezius muscle following needle stimulation (acupuncture) between healthy subjects and patients with fibromyalgia and work-related trapezius myalgia. Eur J Pain 9: 497-510.

Copyright: (C2020 Sheng-Xing Ma. This is an open-access article distributed under the terms of the Creative Commons Attribution License, which permits unrestricted use, distribution, and reproduction in any medium, provided the original author and source are credited. 